\title{
Relationships between photospheric magnetic field, solar wind and geomagnetic perturbations over last 30 years
}

\author{
Elena Gavryuseva \\ Istituto Nazionale di Astrofisica - Arcetri Astrophysical Observatory, \\ Largo Enrico Fermi 5, I-50125 Florence, Italy \\ email: elena@arcetri.astro.it
}

\begin{abstract}
The main aim of this research is to make direct comparison between the basic structure and dynamics of the photospheric magnetic field, solar wind and geomagnetic characteristics without using theoretical assumptions. The heliospheric and geomagnetic data are found to be divided into two groups characterized by their response to the variability of the latitudinal structures of the photospheric field on short and on long time scales.
\end{abstract}

Keywords. Sun: magnetic fields, corona, solar wind, interplanetary medium

Global magnetic field topology has a great influence on the coronal field, heliospheric structure and geomagnetic perturbations. Direct comparison was performed between the temporal behavior of the mean latitudinal photospheric field, heliospheric and magnetospheric characteristics to reveal their relationships and to search for solar drivers at different heliolatitudes during the last three cycles of activity without using theoretical assumptions, models, possible physical expectations, etc.

We use Wilcox Solar Observatory (WSO) data of the large scale photospheric magnetic fields (SMF) available at http://wso.stanford.edu/synoptic.html, Scherrer (1977).

The solar wind (SW) and geomagnetic (GM) data were taken from the OMNI website (http://nssdc.gsfc.nasa.gov/omniweb) which contains the daily mean and the Bartels (BT) mean values of the interplanetary characteristics measured by various space crafts near the Earth's orbit, as well as geomagnetic and solar activity indices. OMNI data include an interplanetary magnetic field (IMF) $B$, its components $B_{x}, B_{y}, B_{z}$, temperature $T_{p}$, pressure $P$, electron $\left(N_{\beta}\right.$ and proton $N_{p}$ density, speed $V_{p}$, electric field $E, N_{\alpha} / N_{p}$ ratio, Mach number $M_{a}$, at the Earth's orbit, indices of geomagnetic (GM) activity $K_{p}$, $A E, D S T$ and the sunspot number $S S N$ for the period 1976-2004.

The same filters and methods have been applied to the WSO and OMNI data to select their long and short term variability in the interval from 1.5 to 25 years about. Yearly variability due to the inclination of the Earth orbit was removed by the 1-year smoothing.

The latitudinal structure was revealed by the analysis of the distribution of the SMF mean over one or more rotations (see, for example Scherrer (1977), Ulrich (2003), Gavryuseva (2006), and the contributions by Gavryuseva to this proceedings). The photospheric field topology composed of the large scale four zonal latitudinal $(4 Z-)$ structure with a 22 -year period with boundaries of the polarity zones located at 25 South, 0, and 25 North degrees and the waves of the magnetic field running through latitudes and changing the polarity with a period of 2-3 years ( $W M F$-topology).

The IMF, SW and geomagnetic variability was studied by different authors. It was noted (see, for example Kane (2005a,b) and Rivin( 1989) that solar indices the geomagnetic field exibit the biennial variations modulated by a 10 -year period. This well agrees 
with the presence of the running wave topology in the photospheric field, which varies the magnetic field with a periodicity of quasi 2.5 years and interferes with the 4-zonal structure changing with the 22-year period.

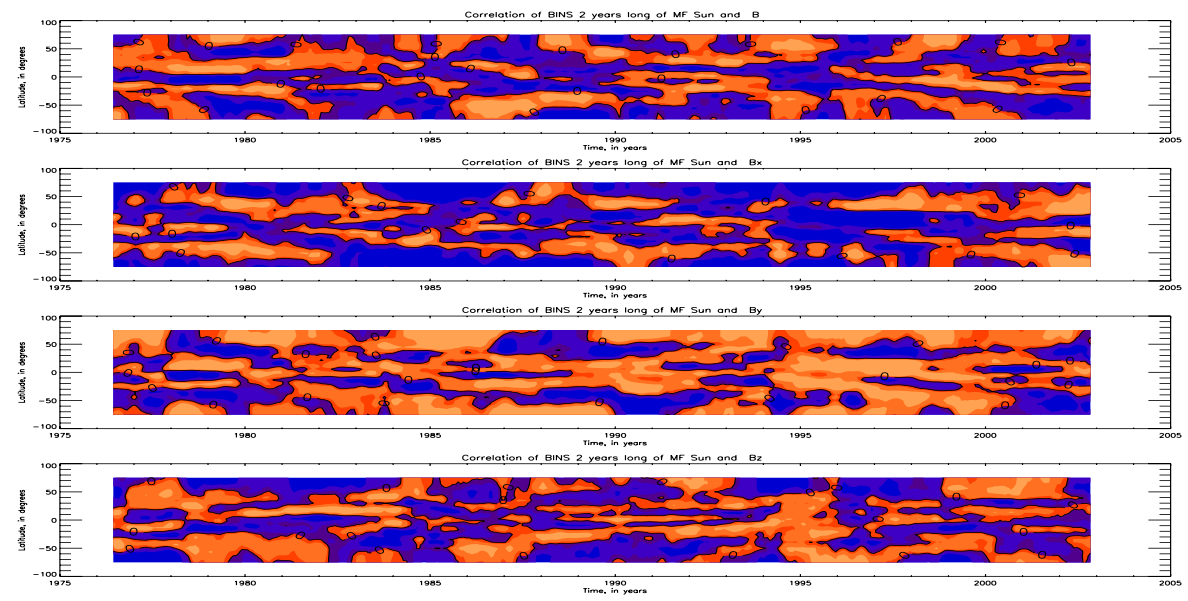

Figure 1. Correlation between the 2-year long sub-sets of the photospheric field with the interplanetary magnetic field intensity $B$ and components $B_{x}$ (upper plot), and components $B_{x}, B_{y}$ (middle plots) and $B_{z}$ (bottom plot) as a function of time and latitude. Orange and blue (light and dark in white-and-black version) colors indicate positive and negative correlation coefficient values.

Correlation between the IMF and the SMF at different latitudes shows that the interplanetary field follows to the variability of the photospheric magnetic field with an optimal delay of about 4 days. This delay is used in the calcullations of the correlation between the sets of the Bartels means of the SMF and OMNI data.

In Fig. 1 the correlation coefficients between the SMF, intensity $B$ and components $B_{x}, B_{y}$ and $B_{z}$ of the IMF are plotted from the top to the bottom for the 2-year long sub-sets of the SMF and IMF data running in time with $1 \mathrm{CR}$ step. The correlation between the SMF and $B_{x}$ component, and between the SMF and $B_{y}$ component is of opposite sign on a long time scale (due to the choise of thye coordinate system). This fact could be used to verify and to confirm conclusions related to one of them. Strong correlation (anti-correlation) does exist between the photospheric SMF on the latitudes above the 50 degrees and $B_{y}\left(B_{x}\right)$ component during minima of solar activity which took place around 1976-1977, 1985-1986 and 1995-1996. The correlation reaches the level of 0.92 .

The $B_{z}$ component correlates to the pre-equatorial SMF in the northern hemisphere and middle latitude zone in the southern hemisphere during maximum of cycle No 21 , it is opposite to its behavior in cycle No 22 and similar in cycle No 23, but additionally at the maximum of the last cycle $B_{z}$ correlates to the sub-polar SMF in the northern and in the southern hemispheres. Generally speaking the interplanetary field was well synchronized with the photospheric field variability in the broad region of latitudes in cycle No 23: this means that the low-latitude solar wind originated from isolated lowlatitude and mid-latitude coronal holes could contribute significantly during the cycle. This finding contradicts the paradigm of a solar wind source consisting of two polar outflows only flanking a planar current sheet Pizzo (1982), but is in agreement with the results of Wang et al. (2000) demonstrating that around solar minimum the sources of the Sun's open magnetic field whose extension is the IMF are the big polar coronal holes, 

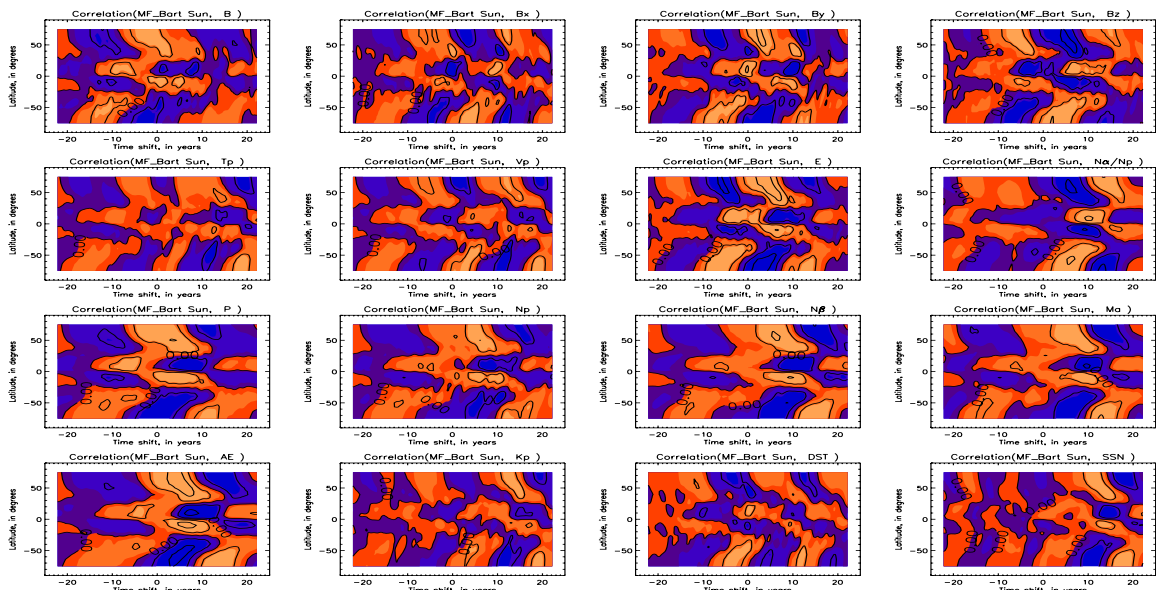

Figure 2. Correlation coefficients of the photospheric field (taking into account its polarity) with solar wind characteristics and geomagnetic perturbations as a function of delay in years and of latitude. Orange and red (blue) colors indicate positive (negative) correlation coefficient values.

while at solar maximum these are the small low-latitude coronal holes but, as we can see, not only they (see, for example Gonzalez et al. (1994, 1999); Wang et al. (2000); and references there).

Correlation coefficients $K_{\text {cor }}$ between full data sets from 1976 to 2004 with one Bartels rotation resolution of the photospheric field from one side and of the solar wind and geomagnetic characteristics (OMNI data) on the other side have been calculated for different latitudes with the shift up to 22 years. Fig. 2 shows these correlation coefficients
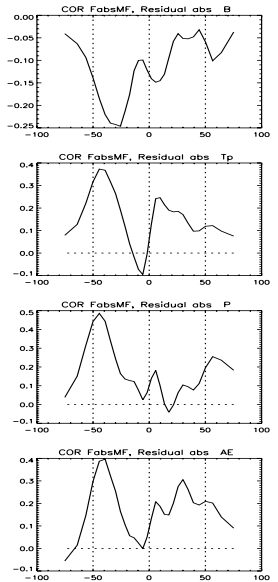
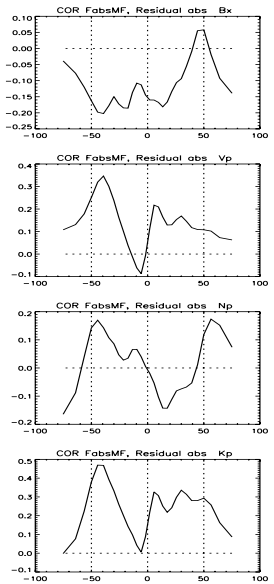
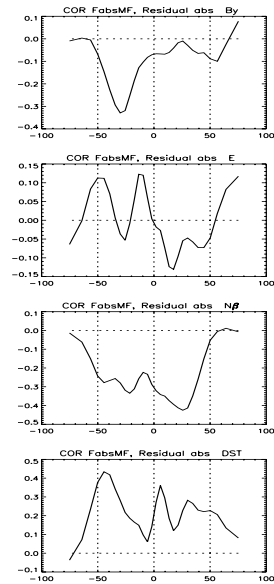
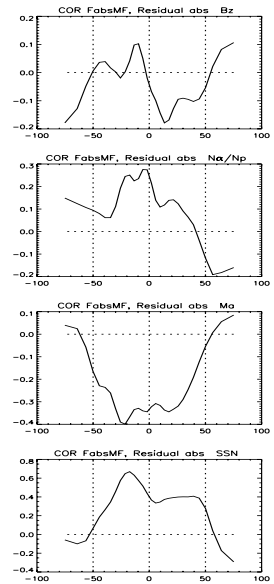

Figure 3. Coefficient of correlation between short term variabilities of the photospheric field at different latitudes and the IMF, solar wind and geomagnetic characteristics.

$K_{\text {cor }}$ smoothed by a 1-year running window as functions of latitude and time delay between the SMF and the interplanetary field intensity $B$ and $B_{x}, B_{y}, B_{z}$ components (upper plot), and then down to the bottom for $K_{\text {cor }}$ between the yearly means of the SMF and $T_{p}, V_{p}, E, N_{\alpha} / N_{p}$ ratio (plots in second line); $P, N_{p}, N_{\beta}$, Mach number $M_{a}$ (plots in third line); $A E, K_{p}, D S T$ and the sunspot number $S S N$ (plots in bottom line). The data used take into account the sign of the characteristics. 
The latitudinal dependence of these correlations is due to the 4-zone SMF topology. The correlation between the SMF and $B_{x}, B_{y}$ and $B_{z}$ components has a 22-year periodical behavior due to the 22-year variability of the solar magnetic field. The correlation coefficients $K_{\text {cor }}\left(S M F, B_{y}\right)$ is anti-correlated to the correlation coefficients $K_{c o r}\left(S M F, B_{x}\right)$ and $K_{c o r}\left(S M F, B_{z}\right)$ between the SMF and $B_{x}$ and $B_{z}$ components. The relationship between the photospheric field and $E$ is similar to the one between the SMF and $B_{y}$, while the $K_{c o r}\left(S M F, T_{p}\right)$ and $K_{c o r}\left(S M F, V_{p}\right)$ are similar to the $K_{c o r}\left(S M F, B_{x}\right)$, and opposite to the $K_{c o r}\left(S M F, B_{y}\right)$. And at the end $K_{p}$ has the same relationship with the $\mathrm{SMF}$ as $B_{x}$ component of the IMF and opposite to th $K_{\text {cor }}(S M F, D S T)$.

The correlation between the SMF and the intensity $B$ shows a quasi 30-year periodicity. The relationship between the photospheric field and $P, N_{p}, N_{\beta}, M_{a}$ is similar, while $K_{\text {cor }}\left(S M F, N_{\alpha} / N_{p}\right)$ has an opposite sign. The correlation coefficient $K_{c o r}(S M F, A E)$ is similar to the $K_{c o r}(S M F, B)$. Comparison of the $K_{c o r}(S M F(\theta, t), O M N I(\theta, t))$ shows that the OMNI data are divided into two groups which demonstrate similar dependence of the $K_{\text {cor }}(\theta$, tshift $)$ on the time shift between the $\operatorname{SMF}(\theta, t)$ and $\operatorname{OMNI}(\theta, t)$.

The first group is composed of $B, P, N_{p}, N_{\beta}, M_{a},-N_{\alpha} / N_{p}, A E$ and $-S S N$. The second one includes $B_{x},-B_{y}, B_{z}, T_{p}, V_{p},-E, K_{p}$ and $-D S T$. The positive value of $K_{c o r}$ is an indicator of a possible connection between the SMF and a SW, but it is not enough to conclude that the SMF and a SW are physically dependent.

Since quasi biennial variability is present in the solar magnetic field it is necessary to verify the physical relationship between the variability of the SMF, IMF, solar wind and geomagnetic data on a short term scale of about 2-3 years. An analysis of the relationship of the short term variability of the solar magnetic field and SW data has been performed to complete this study.

The latitudinal dependences of the $K_{c o r}(F M F, F S W)$ were found for the correlation between the residuals of the photospheric magnetic field (FMF) with the residuals of the different SW data (FSW) calculated as difference between yearly and 4-year means of the SMF and SW data and presented in Fig. 3.

Coefficients of correlation with the optimal delay between the short term variabilities of the photospheric field at different latitudes and the interplanetary magnetic field, solar wind and geomagnetic characteristics have been calcullated and the same two groups could be selected in the OMNI data from the point of view of their relation with the photospheric field. In this way it was established that there are two groups of the interplanetary data led by the $B$ and by the $B_{x}$ (or $-B_{y}, B_{z}$ ) which respond to the variability of the photospheric magnetic field in a similar way (from the point of view of the periodical character and of the latitudinal dependence of the $K_{c o r}(S M F, S W)$ or the $K_{c o r}(F M F, F S W)$ and they have a similar response in the geomagnetic perturbations of $A E, K_{p}$ and $-D S T$ indices). Physical connections between the Sun and the interplanetary characteristics could be attributed to the influence of the intensity of the solar magnetic field (absMF) without taking into account its polarity. The corresponding correlation coefficients between the 1-year mean values of the SMF intensity and absolute values of the SW data $(a b s S W)$ as functions of time delay in years and in latitude are shown in Fig. 4. The $K_{c o r}(a b s M F, a b s S W)$ have an 11-year periodicity for all the SW data except the IMF intensity $B$, and slightly visible in the $K_{c o r}$ for the absolute values of the $B_{y}: a b s B_{y}$ and $A E: a b s A E$.

There is a remarkable particularity of the latitudinal dependence of the coefficieent of correlation $K_{c o r}(a b s M F, a b s S W)$ : the change of a sign and a phase shift of the correlation coefficients at the level of about 50-55 degrees. This fact could be interpreted in terms of the magnetic field lines open to the space and do not approuching the Earth orbit where the OMNI data were taken. The $K_{c o r}(a b s M F, a b s S W)$ are symmetric to 

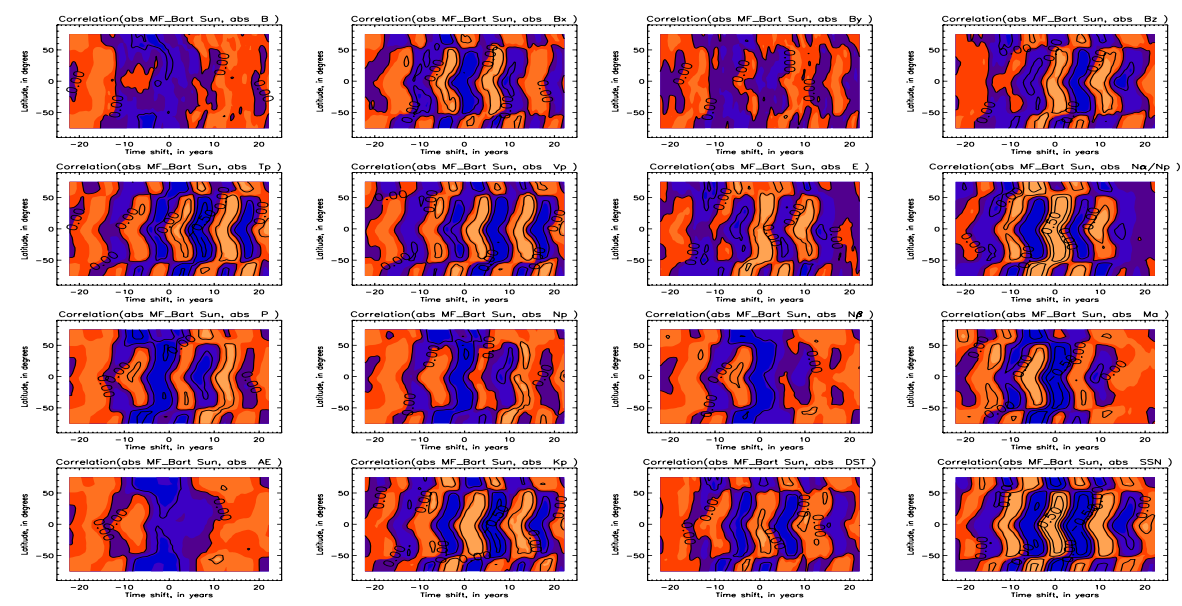

Figure 4. The correlation coefficients of absolute values of the photospheric field with absolute values of solar wind and geomagnetic characteristics as a function of delay and latitude.

the equator. This is well illustrated by Fig. 4 for the latitudinal dependence of the $K_{\text {cor }}(a b s M F, a b s S W)$ for the fixed optimal delay between the SMF intensity and the absolute values of the SW data. This figure is analogous to Fig. 2 (for the real SMF and SW values) where the $K_{c o r}(M F, S W)$ are antisymmetric to the equator. There is a clear anti-correlation between the intensity of the photospheric field of the activity belts and $B, V_{p}, P, N_{p}, N_{\beta}$ and $M_{a}$. This confirms that during high activity periods the slow solar wind prevails. Positive correlations of the abs $M F$ with the absolute values of the $A E, K_{p}$ and $D S T$ indices correspond to the statement that most of the geomagnetic perturbations are originated from the low and middle latitudes when the intensity of the magnetic field is high. The difference between Fig. 2 and Fig. 4 permits to investigate the sensitivity of the SW characteristics to the SMF polarity (and not only to the intensity of the SMF) and to the basic topology of the solar magnetic field.

\section{Some summary remarks}

Southward-directed a primary cause of geomagnetic perturbations Durney (1961); Gonzales et al. $(1994,1999)$. In this way the orientation of the interplanetary magnetic field Axford and McKenzie (1997); Low (1996); Parker (1997); Smith (1997) plays an important role. The intensity and the frequency of of solar activity phenomena depend on the sunspot cycle, which can be characterized by the variability of the SMF intensity in time and over the latitudes. The topology of the solar magnetic field influences the geomagnetic perturbations through the intensity and orientation of the interplanetary magnetic field and/or through other characteristics of the solar wind. In this approach we could understand the presence of two groups of the OMNI data similarly sensitive to the basic topology of the magnetic field of the Sun (from point of view of the dependence of the coefficients of their correlation with the mean latitudinal magnetic field on the latitude and phase shift).

Useful information was deduced from the $K_{c o r}$ distribution for the correlation between the SMF and different characteristics. We found that the photospheric field influences the magnitude of the interplanetary field and, in the same way, the proton density, flow pressure, Alfven Mach number and plasma beta respond to the SMF. The $A E$-index behaves in a similar way to these solar wind characteristics. 
On the contrary, regarding the planetary geomagnetic activity index $K_{p}$ we can deduce the following chain. Solar activity events (CME, magnetic field intensity, sunspots, etc.) through perturbations of the $B_{z}$ component $\left(B_{x}, B_{y}\right.$ components) of the IMF, the proton temperature $T_{p}$, plasma speed $V_{p}, N_{\alpha} / N_{p}$ ratio influence the $K_{p}$ index. The variations of the $-B_{z}\left(B_{y}\right)$ component produce the perturbations of the DST index, which are of opposite sign to the $K_{p}$ and $B_{x}$ time dependence.

These results are useful for the understanding of the origin of solar wind and geomagnetic perturbations and for long term predictions. The formal and complete approach taken here to the problem of solar-terrestrial relations reveals the physical connections between the processes on the way from the Sun to the Earth.

\section{Acknowledgements}

I am grateful to the WSO and OMNI teams for making available data of measurements of the solar magnetic field, solar wind and geomagnetic characteristics. I am deeply grateful to Prof. G. Godoli for his stimulating interest in these results, and help in preparation of this paper. I am very grateful to Prof. B. Draine for useful advises and great help.

\section{References}

Durney, J.W. (1961), Phys. Rev. Lett., 6, 47.

Axford, W.L. \& McKenzie J.F. (1997), in Cosmic winds and the heliosphere, ed. by J.R. Jokipii, C.P. Sonett \& M.S. Giampapa, p. 31.

Gavryuseva, E., \& G. Godoli (2006), Physics and Chemistry of the Earth, Elsevier. 31, 68

Gavryuseva, E. (2006a), News of the Academy of Science, IzvRAN, ser. Physics, 70, No. 1, 102.

Gonzalez, W.D., J.A. Joselyn, Y. Kamide, H.W. Krorhl, G. Rostoker, B.T. Tsurutani \& V.M. Vasyliunas (1994), J. Geophys. Res., 99, 5771.

Gonzalez, W.D., B.T. Tsurutani \& A.L. Clùa de Gonzalez (1999), Interplanetary origin of geomagnetic storms, Space Science Rev., 88, 529.

Kane, R.P. (2005a), J. Geophys. Res., 110, A01108.

Kane, R.P. (2005a), Solar Phys., 227, 155.

Low, B.C. (1996), Solar Phys., 167, 217.

Parker, E.N., (1997), in Cosmic winds and the heliosphere, ed. by J.R. Jokipii, C.P. Sonett \& M.S. Giampapa, 3.

Rivin, Yu.R. (1989), Nauka, IZMIRAN, 36.

Smith, E.J., (1997), in Cosmic winds and the heliosphere, ed. by J.R. Jokipii, C.P. Sonett and M.S. Giampapa, 425.

Scherrer, P.H., J.M. Wilcox, L.Svalgaard, T.L. Duvall, Ph.H. Dittmer \& E.K. Gustafson (1977), Solar Phys., 54, 353.

Wang, Y.-M., J. Lean, \& N. R. Sheeley (2000), The long-term variation of the Sun's open magnetic flux, Geophys. Res. Lett., 27, 505. 